\title{
Geometrical Analysis of a Continuously Variable Transmission System Designed for Human- Robot Interfaces
}

\author{
Emir Mobedi and Mehmet İsmet Can Dede
}

Mechanical Engineering Department, İzmir Institute of Technology, 35430 İzmir, Turkey

\begin{abstract}
New robotic systems are placed out of their constrained workspaces in order to work alongside humans. Consequently, these applications call for robots monitoring and regulating physical human-robot interaction. These robots' mechanical compliance should be varied when they are in physical contact with the human or their changing environments. This compliance variation can be achieved in a variety of ways. However, one common idea is the variation of joint stiffness mechanically, electromechanically or by control. The solution presented in this paper is an electromechanical way of varying the joint stiffness. Among the electromechanical methods for varying the joint stiffness, continuously variable transmission (CVT) systems can be used in human-robot interfaces if a set of design criteria are met. These criteria include backdrivability, independent output position and stiffness variation, shock absorbing and low mass/inertia. In this paper, a novel two-cone CVT design with a double spherical transmission element is introduced by taking into account the abovementioned criteria. Additionally, design parameters are identified via carrying out a geometrical analysis of this new CVT system.
\end{abstract}

Keywords: Continuously variable transmission, Variable stiffness actuation, Human-robot interface

\section{Introduction}

The first robot applications in the industry required only motion control strategies. The first examples of these robots are in the automotive industry and are employed for pick-and-place, painting and welding jobs. The main function of these robots is to accomplish these tasks by following a predefined motion trajectory with minimum errors. The major disadvantage of these conventional industrial robots is that they cannot be used in unpredictable environments such as working next to human coworkers. Even if motion control is achieved with high accuracy, the safety factor should be considered in case of a collision with a human coworker. Hence, these robots are confined in well-defined and constrained working cells in which they can only operate when there is no human coworker is inside.

In recent years, a new generation of robots, known as human-robot interfaces, have been developed to work alongside humans not only in industrial settings but also in households. The well-known examples are industrial coworkers, household robots, advanced prostheses and haptic devices (Samur, 2012). These new generation robots differ from the conventional ones in their performance criteria. While accuracy and repeatability are the key metrics of performance in conventional robots, the key metrics in these robots include easiness of backdriving the robot by physically interacting with its end-effector (also known as backrivability) and stiffness variation range (also known as impedance width) are the prominent ones.

As an example of robots collaborating with humans, haptic devices display varying mechanical impedances to the human operator by regulating the amount of forces felt against the motion induced by the human operator. Since a haptic device should display a wide range of impedance values, one of the performance metrics of a haptic device is determined by this range. The minimum impedance is the resistance that the haptic device displays against motion when the desired resistance to be displayed is zero. The maximum impedance is the maximum applicable resistance that can be displayed against the motion of the human. Some factors affecting these measures are the frictions at the joints of the device 
and inertia of the links. While the increase in the value of these factors affects minimum impedance adversely, the maximum impedance performance is improved. Therefore, haptic device design is accomplished by adjusting the trade-off between the minimum and maximum impedance characteristics of the device. However, it is the variability of the joint stiffness that moves the device performance from a minimum impedance range to a maximum impedance range. Commonly, this is achieved by employing a suitable controller to regulate the actuator's output torque with respect to the motion induced by the human (Samur, 2012). While the range of the impedance is dependent on the motor's capability up to a certain level, variable stiffness joint structures are designed to increase this impedance range.

Another cluster of robots in the field of human-robot interaction is collaborative industrial robots. These robots are designed to work alongside human coworkers. They are developed in compliance with the ISO 10218-1 standard in order to prevent any damage to the human coworker. First clause of this standard (5.10.2) defines a condition that no motion will be allowed if the human coworker is in the collaborative workspace. The working condition stated in the second clause (5.10.3) explains that the robot can be moved as long as the human coworker applies direct force input to the robot. The other clause (5.10.4) is related to the distance defined between the human coworker and the robot. The operation can be carried out if the minimum distance defined between the robot and the human coworker is not violated. In the final clause (5.10.5), robots with inherent force limitations may work alongside the human coworker in continuous contact because they are not capable of applying excessive forces to the human coworker. This force limitation can be achieved by control, mechanical design or a combination of both.

The main difference of collaborative robots with respect to conventional ones is that pure motion control is not sufficient to achieve new tasks anymore since physical interaction takes place between the human and the robot. Thus, they should comply with their environment in order to execute their tasks safely. In other words, these robots should not apply excessive amounts of force in the case of physical contact with their environment. If the robot interacts with a stiff wall, the environment stiffness will be higher. On the other hand, if the physical interaction happens with a human, the environment stiffness is lower. Since in a general application scenario the robot is expected to physically interact with different objects with different stiffness characteristics, the robot's joint stiffness should be adjusted as the environment stiffness changes. Similar to human anatomy, these robots' joint stiffness should be controlled. Joint stiffness control can be achieved either actively or passively (Migliore et al., 2005).

Active joint stiffness control is achieved by implementing compliant control algorithms. Via these algorithms, the impedance of the joint is changed by control (Vanderborght et al., 2013). If high position accuracy is required in some directions of the workspace where there is no physical constraint, stiff actuation with a high-gain controller is preferred. Hence, if some unexpected external forces are applied to the end-effector of the robot, the robot will apply larger forces to compensate for this effect in order to follow the predefined motion trajectory closely. Conversely, if high joint compliance is required in another direction of the workspace in which there is a physical constraint, soft joint actuation with a lowgain controller is employed for the motion along this direction. In this case, if undesired external forces are exerted on the end-effector along one direction, for example when tracking an irregular surface, a low gain controller can be selected for the motion along the normal of the surface. As a result of this, the position error will be higher in that specific direction than the stiff joint actuation position error to prevent significant impacts on the surface. Applying active joint stiffness control has some drawbacks. In these types of closed-loop control system designs, maintaining stability is always a challenge. Also, the selection of the force sensor is important since the stiffness of the force sensor will identify the use of this active stiffness controller for a certain application with a predefined stiffness range.

Passive stiffness control is sub-divided into two groups, mechanical and electromechanical. Passive stiffness control is also classified as inherent compliance (Vanderborght et al., 2013). In purely mechanical stiffness control, fixed compliance is provided by adding either an intrinsically compliant 
element like springs between the end-effector and the actuator or a mechanism providing a constant transmission ratio. Subsequently, a fixed stiffness is obtained which can be used in applications with well-defined environments. The capstan drive system is a common choice of a transmission system for haptic devices which also results in fixed joint stiffness. The main uncertainty of this design is the backlash due to the slip problem (Lu et al., 2012). Furthermore, the fundamental imperfection is that these actuation systems with fixed stiffness do not have the potential to change the stiffness of the joint in the course of operation. Nevertheless, by active control of the actuator, the joint stiffness can be regulated within a predefined limit. Hence, these actuator designs are not suitable for environments that have highly varying stiffness characteristics.

Actuators with adaptable joint compliance have the capacity to interact with dynamic environments with varying stiffness characteristics. A general approach to configure these types of actuators is using an elastic element between the actuator and the joint structure, and continuously controlling the compression/tension of this elastic element via an additional actuator. As a result of this, while the joint's motion is controlled, the actuation system's stiffness can be changed independently. Moreover, these types of actuators have shock absorbing properties due to the existence of an elastic element, which prevents possible physical damage during a significant physical impact.

Among a variety designs for actuators with adaptable joint compliance, in biologically inspired antagonistic springs with antagonistic motors type of joint design, joint stiffness and joint angle are changed independently without using a current controller for the motor actuation (Migliore et al., 2005). Since stiffness is obtained passively, stability is guaranteed. Nevertheless, the joint motion range is limited in this design.

The cross-coupled variable stiffness actuator (VSA), has the capability to work in an unlimited operating range. In this design, a timing belt is continuously in contact with pulleys applying tension, and pulleys are coupled to the motors' shafts (Tonietti et al., 2005, and Vanderborght et al., 2013). As in any application with timing belts working under tension, this part should be replaced after a duration of use.

In another design called bidirectional antagonism with variable stiffness (BAVS), a harmonic drive is used as the transmission system, which may result in obtaining higher torque outputs. In this design, the output shaft is connected to the handle with the help of a pinion crown wheel system. This system's disadvantage is that the system does not have the capability to absorb shock due to an unexpected impact (Ishida et al., 2006). Further, the pinion crown wheel system adds an extra inertia to the mechanism. Hence, it affects the backdrivability adversely.

Antagonistic springs with independent motors have shock-absorbing features due to the spring located between the joint and base. Furthermore, this design is smaller in size with respect to the previous designs. Hence, it has the capacity to be utilized in wider range of applications (Wolf et al., 2008, and Grebenstein et al., 2011). On the other hand, since the stiffness motor is located on the joint, the total moving inertia is increased. Therefore, the backdrivability property is adversely affected.

Additionally, the lever length type of variable stiffness joint design also has a shock-absorbing feature and independent position-stiffness variation property (Visser et al., 2011). However, the mechanism occupies a larger volume than the other VSA designs, and this limits the application of this system in human-robot interfaces.

Alternative to the above approaches, continuously variable transmission (CVT) systems have been used to vary the stiffness of the joint continuously by changing the transmission ratio continuously. These systems have been widely used in automotive transmissions. In an ideal condition, these systems have the potential to provide continuously varying power transmission within a predefined limit. This transmission can be accomplished via friction, belt or gear systems (Sclater et al., 2001). One example of gear-based CVT systems are planetary gear based CVT systems (Ivanov et al., 2012). However, they 
are not capable of varying the stiffness independent of the output movement since the input, which is needed to increase the output torque, is supplied from the external load.

Another type of CVT is the toroidal traction drive (Novellis et al., 2012). In this drive system, there are input and output discs, which are constructed to form a toroidal cavity. In addition, a suitable number of rollers are used as the transmission element. By steering the rollers that are contacted continuously with input and output discs, the output speed is regulated. The main principle of this speed adjustment is based on the difference between the effective radius of the input and output discs. To increase the upper level of transmissible torques and speeds, a specific lubricant is used at the contact points under $3 \mathrm{GPa}$ pressure (Carbone et al., 2004). Different types of toroidal traction drives have been developed including singe-roller half toroidal variator, single-roller full toroidal variator, and double roller full toroidal variator. The main application areas of these transmission systems are identified as highway systems, larger engine cars, trucks (Carbone et al., 2004) and industrial machines (Yildiz et al., 2015).

In addition to the abovementioned designs, flywheel-infinitely variable transmission systems are constructed to be used in the construction of an exoskeleton robot (Alò et al., 2018). In this case, a flywheel with the infinitely variable transmission (IVT) is coupled to a human knee in order to assist the human during walking. The significant feature of this design is that since flywheel stores and releases the energy during walking, the necessary amount of energy to be supplied from the motor is reduced. Thus, a smaller motor can be selected for this design.

A different version of IVT has been developed to alter the output stiffness via friction force. The working principle is the same as friction-based CVT cone-drive systems. This design is used in a haptic device design (Faulring et al., 2007).

CVT based variable stiffness mechanisms can find application in a human-robot interface if the specific design criteria of backdrivability, independent output position and stiffness variation, shock absorbing and low mass and inertia are met. Although there are various CVT designs in the literature for human-robot interfaces (Kim et al., 2002; Faulring et al., 2007), the primary limitation of the two-cone drive CVT designs is that the transmission ratio and the output position cannot be altered independently. The source of this problem is that the friction wheel, which is designed to transmit the torque from the input cone to the output cone, should be moved along a linear path to vary the transmission ratio. This gives rise to remarkable longitudinal friction force along the linear path.

In this study, we propose a new variable stiffness actuation system to be used in human-robot interfaces. This system is based on two-cone friction drive CVT, which provides a solution for the independent transmission ratio and motion variation problem. The solution is formulated by using spherical transmission elements instead of the friction wheel. The presented design also aims to guarantee bidirectional power transmission and allows adjustment of shock absorption characteristics. The working principle of the novel CVT design is presented in the next section. The geometrical analysis of the design is carried out to identify the design parameters. The analysis results are validated by using the model of the CVT in a computer-aided design (CAD) environment. Obtained results are discussed and the work is concluded in the last section by addressing future work.

\section{Working principle of the Novel CVT}

In this section, the working principle of the novel CVT and the design details are presented. In conventional friction-drive two-cone CVTs are two cones, which are covered with friction material, and a transmission wheel are used to transmit the moment from the input shaft to the output shaft. These input and output shafts are coupled to respective cones. In order to vary the transmission ratio, the transmission wheel is moved along a straight path while the effective radii measured at the contact points of the cones are changed. However, it is not possible to control the output position and transmission ratio independently in this way since the transmission wheel does not have the capability to perform the 
holonomic motion. In other words, the cones must be rotated to ease the change of the transmission ratio, and this condition limits the usability of the two-cone CVT drive in human-robot interfaces.

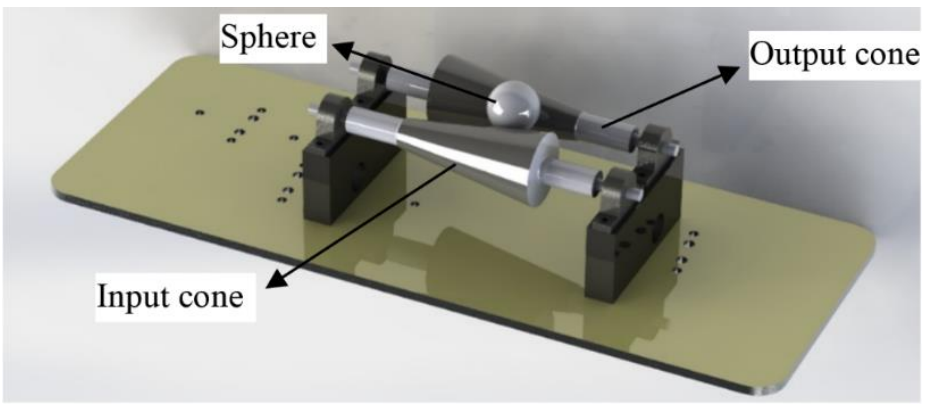

Figure 1. Isometric view of the single-sphere two-cone CVT (Mobedi and Dede, 2018)

In order to ensure holonomic motion, the transmission wheel is swapped with a spherical transmission element (Figure 1) which will be called the sphere from this point on. Hence, in a rigid body approach, point type of contact with the two cones is guaranteed, allowing the control of output position and transmission ratio independent from each other. In other words, stiffness variation with constant load and stiffness variation at constant position requirements, which are stated in (Wolf et al., 2016), can be achieved with this transmission system. The cones are covered with a friction material to create friction surfaces on the cones to avoid slippage without applying a larger amount of normal force on the cones. A diaphragm or any other material a with higher friction coefficient can be chosen for constructing this friction surface.

The location of the sphere along the normal of the plane formed by the rotation axes of the cones is important for the linear shifting action during the change of the transmission ratio. If the sphere is placed so that its center is exactly on the plane formed by the rotation axes of the cones, it becomes impossible to change the transmission ratio without changing the output position. This problem is visualized by the help of a sketch drawing presented in Figure 2.

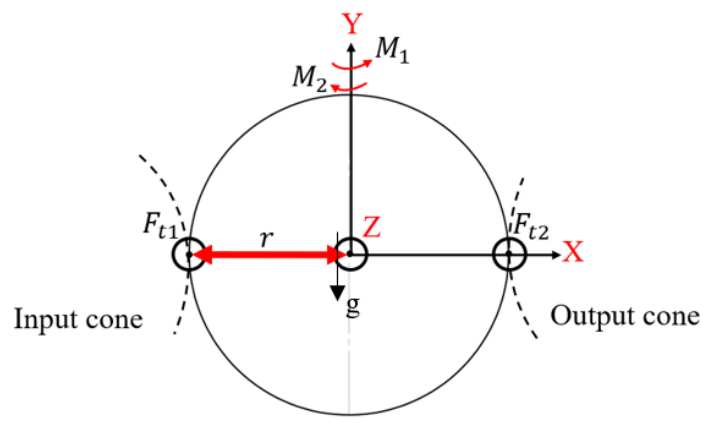

$F_{t 1}$ : Tangential force applied by the input cone

$F_{t 2}$ : Tangential force applied by the output cone

$r$ : Radius of the sphere

Figure 2. The free-body diagram of the sphere located between the cones

$$
\begin{aligned}
& M_{1}=F_{t 1} r \\
& M_{2}=F_{t 2} r
\end{aligned}
$$

When the sphere is translated along the (-)Z-axis direction due to the friction surfaces, tangential friction forces act on the sphere resisting the motion. These tangential forces are equal to each other since the normal forces and friction coefficient are the same for the two cones. Consequently, $M_{1}$ and $M_{2}$ have equal magnitudes as presented in Equations 2.1 and 2.2. However, these moments are in opposite directions, which constrain the sphere to translate without rolling about Y-axis. Hence, the sphere should slip on the cones when changing the transmission ratio. Nevertheless, slip between the cones and the 
transmission element is not desired in order to guarantee the transmission within the selected torque transmission limits. For this reason, while the sphere is translated to vary the transmissions ratio, it should be rolling instead of slipping. In order to formulate a solution for this, the sphere is located above the cones. The mathematical proof of this solution is formulated by Equations 2.3 and 2.4 based on the freebody diagram of the sphere when it is placed above the cones, which is presented in Figure 3 for an arbitrary location of the sphere along the Z-axis.

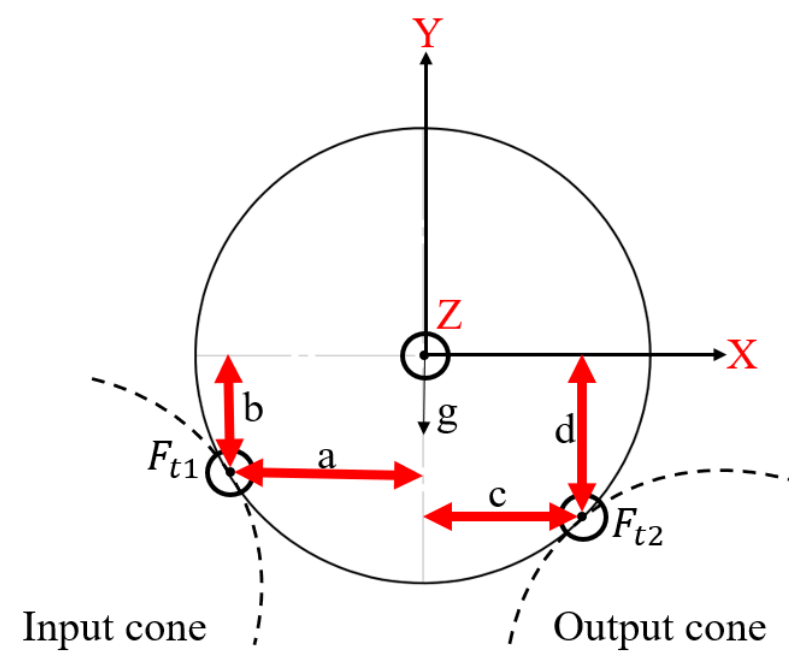

Figure 3. The free-body diagram of the sphere located above the cones

$$
\begin{aligned}
& \sum M_{Y}=F_{t 1} a-F_{t 2} c \\
& \sum M_{X}=-F_{t 1} b-F_{t 2} d
\end{aligned}
$$

When Equations 2.3 and 2.4 are investigated, it is obvious that resultant moment about the X-axis make the sphere roll on the cones and move the sphere in the desired (-)Z-axis. The moments of the tangential forces about the $\mathrm{Y}$-axis act against each other which almost prevent the rotation about the $\mathrm{Y}$-axis. Therefore, with this solution, it is possible to move the transmission element without the necessity of a pure slip condition. This also indicates that the mechanism is capable of adjusting the transmission ratio by minimally disturbing the output position of the cone.

Nevertheless, this relocation of the sphere constitutes new problems. In order to explain these new problems, Figures 4 and 5 are presented. In Figure 4, it is assumed that there is a handle coupled to the output cone and the user holds it and applies a force denoted as "User force" in the figure. When the input torque is applied in the clockwise (CW) direction, the transmission is achieved as indicated in Figure 4. The friction forces acting on the sphere during transmission are in the downward direction $\left(\mathrm{P}_{1}\right.$, $\mathrm{P}_{2}$ ). Hence, transmission without slippage is guaranteed with a higher limit of torque transmission. For the other case in which the input is in the counterclockwise $(\mathrm{CCW})$ direction as presented in Figure 5, torque transmission with the previously defined upper limit cannot be guaranteed since the friction forces acting on the sphere $\left(\mathrm{P}_{3}, \mathrm{P}_{4}\right)$ are upward, reducing the normal force between the sphere and the cones ("W" is the weight of the sphere). This situation results in slippage at the point of contact at lower ranges of transmission torque value. 


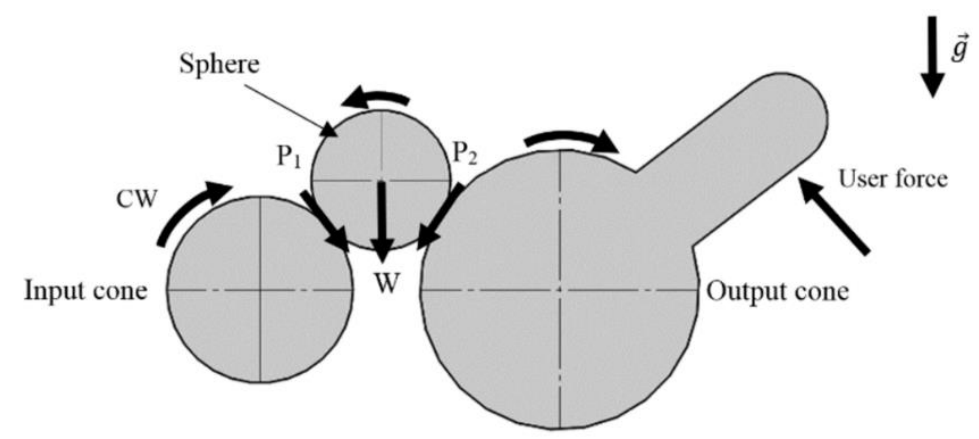

Figure 4. Side view of the one transmission point for CW direction (Mobedi and Dede, 2018)

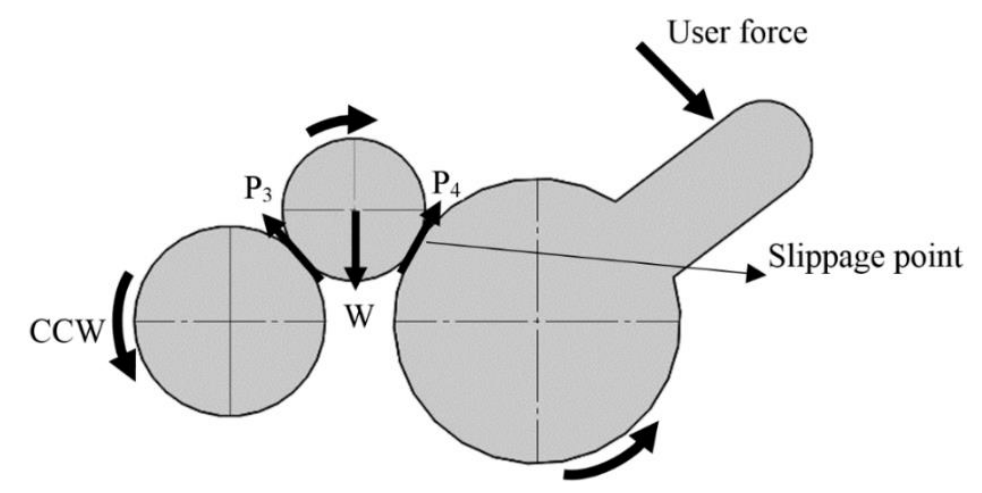

Figure 5. Side view of the one transmission point for CCW direction (Mobedi and Dede, 2018)

In order to propose a solution to this problem, a second sphere below the cones is added to the system. Hence, when one of the cones is in Figure 5 situation, the other will be in Figure 4 situation. Consequently, a constant regulated combined normal force on the cones is guaranteed at all times, which means that the designated torque transmission is achieved independent of the direction of motion. Additionally, as presented in Figure 6, both spheres are pre-tensioned with two additional forces $\left(F_{1}, F_{2}\right)$ guaranteeing the equal amount of normal force acting on each sphere when there is no torque transmission. The calculation of $F_{2}$ in terms of $F_{1}$ and the mass of the sphere, $m$, is stated in Equation 2.5 .

$$
F_{2} \sin \vartheta=m g+F_{1} \sin \emptyset
$$

The isometric view of the double-sphere CVT is presented in Figure 7. In order to validate the working principle, an initial prototype is manufactured and proof of design experiment is conducted to observe if the same amount of torque can be transmitted in both $\mathrm{CW}$ and $\mathrm{CCW}$ directions. This initial experiment validated the performance of the new CVT design, and it was shown that bidirectional power transmission and independent transmission ratio and position variation was accomplished (Mobedi and Dede, 2018). 


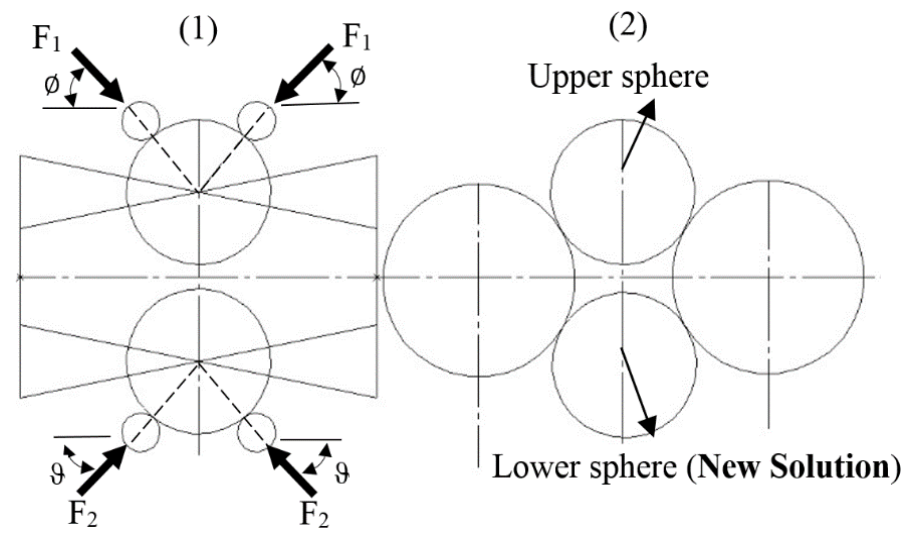

Figure 6. Double-sphere CVT (1) Front view of the system (2) Side view of the system (Mobedi and Dede, 2018)

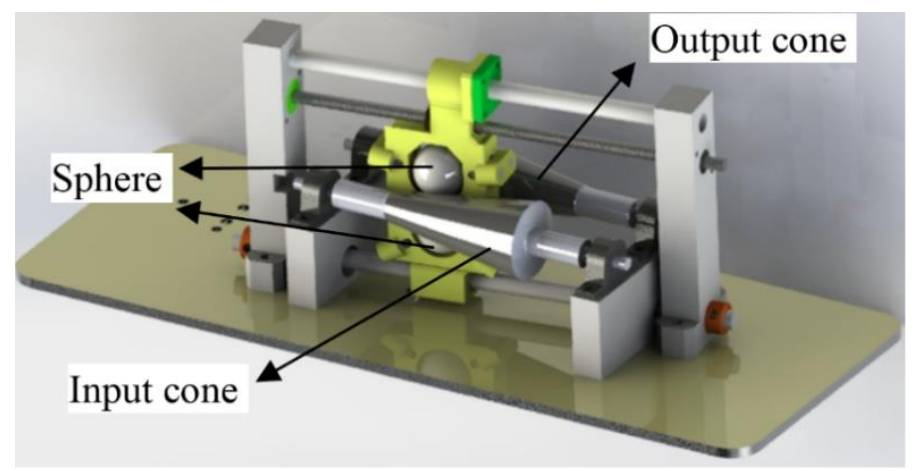

Figure 7. Isometric view of the double-sphere two-cone CVT (Mobedi and Dede, 2018)

In this initial prototype, the spheres are housed inside a carriage system. This carriage system is moved along a linear path to change the transmission ratio. The exploded view of the carriage system is indicated in Figure 8. In this design, there is a total of 8 parts as follows: compression springs (6), set screws (7), support elements $(\mathbf{5})$, four pieces of steel pins $(\mathbf{4})$ and bearings $(\mathbf{3})$, two pieces of steel spheres $(\mathbf{1}, \mathbf{2})$ and the carriage (8). In the assembly process, first, upper and lower spheres are placed into the sphere cavities, and the bearings are placed into the slots. Following this, steel pins are mounted through the bearings' holes so that the spheres can be confined in the carriage. The next step is related to the application of pretension on the spheres. Two support elements are located at the back of the steel pins, and springs are placed behind the aforementioned support elements. Finally, a set-screw (7) is mounted on the spring so that the pretension of the spring can be altered. 


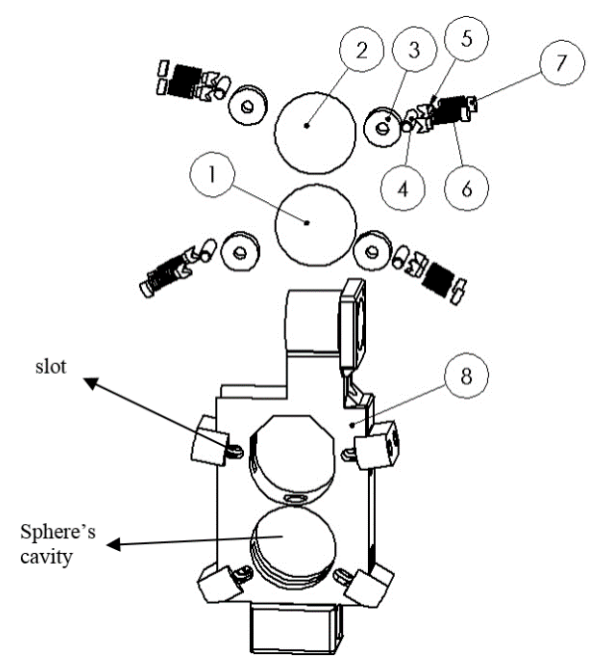

Figure 8. Exploded view of the carriage system (Mobedi and Dede, 2018)

After assembly of the carriage system, the carriage system is assembled on a screw-nut system (11) and linear guides (9) as shown in Figure 9. This screw-nut system is used to move the spheres located on the carriage along the changing radii of the cones. A motor should be coupled to the screw-nut system to move the carriage automatically. The cones are denoted as (10) and the bedding pieces for the cones and the carriage's linear guides are denoted as (12) and (13) respectively in Figure 9.

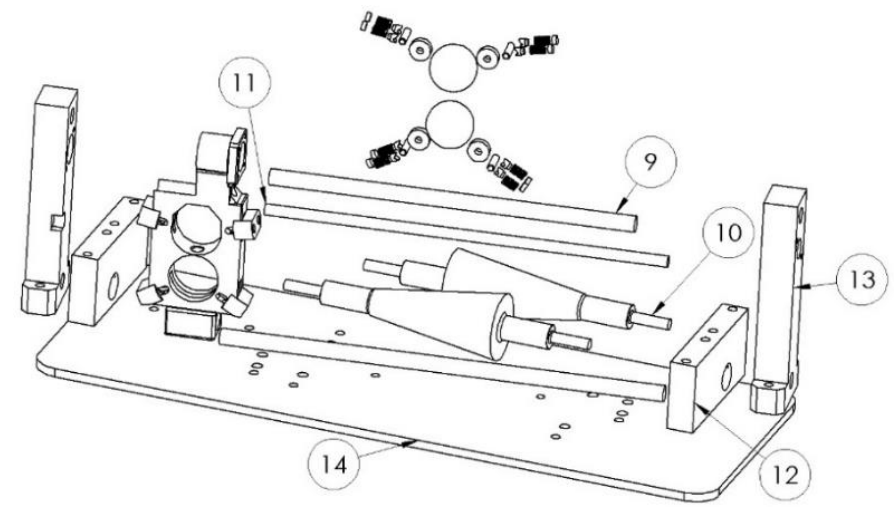

Figure 9. Exploded view of the whole assembly (Mobedi and Dede, 2018)

By adjusting the normal force between the cones and sphere through varying the compression on springs denoted by (6), the transmission torque limits can be set. As an example, if it is required to set the torque transmission limit to lower values, the normal force is reduced by releasing the compression on the spring. As a result of this, when this limit is exceeded there will be slippage between the cones and the sphere. Consequently, an excessive amount of torque to be applied either to the user or to the actuator during operation can be avoided. Therefore, the joint structure is inherently safe in the mechanical design sense.

Bearings denoted with (3) in Figure 8 are used to transmit the compression force to the spheres in order to minimize friction during the linear motion of the carriage. The use of these bearings results in a quicker response time during the transmission ratio variations.

\section{Geometrical Analysis of the CVT}

The primary objective of this section is to present the computations of the positions and orientations of the bodies that compose this CVT design by identifying the design parameters. Rigid body assumption is used in these computations. Through these computations, the limits of the CVT are calculated as a 
function of design parameters so that an optimum design methodology can be applied. To achieve this, the rotations between the frames are defined, and the angles are computed with the help of the plane geometry.

Within the methodology of the geometrical analysis, an arbitrary position of the sphere making contact with the cones is considered. Then, the required planes and triangles are analyzed via the CAD model of the first prototype of the CVT, which is presented in Figure 10. For each defined length or angle, a parametric formula is derived and later compared with the measured values from the CAD model. Afterward, these formulas are coded in Matlab, and the required orientations are calculated symbolically. As a final step, the abovementioned angles are verified with the help of the CAD model of the transmission system created in Solidworks software. The comparison is carried out for five different locations of the sphere indicating different transmission ratios. The results are presented at the end of this section.

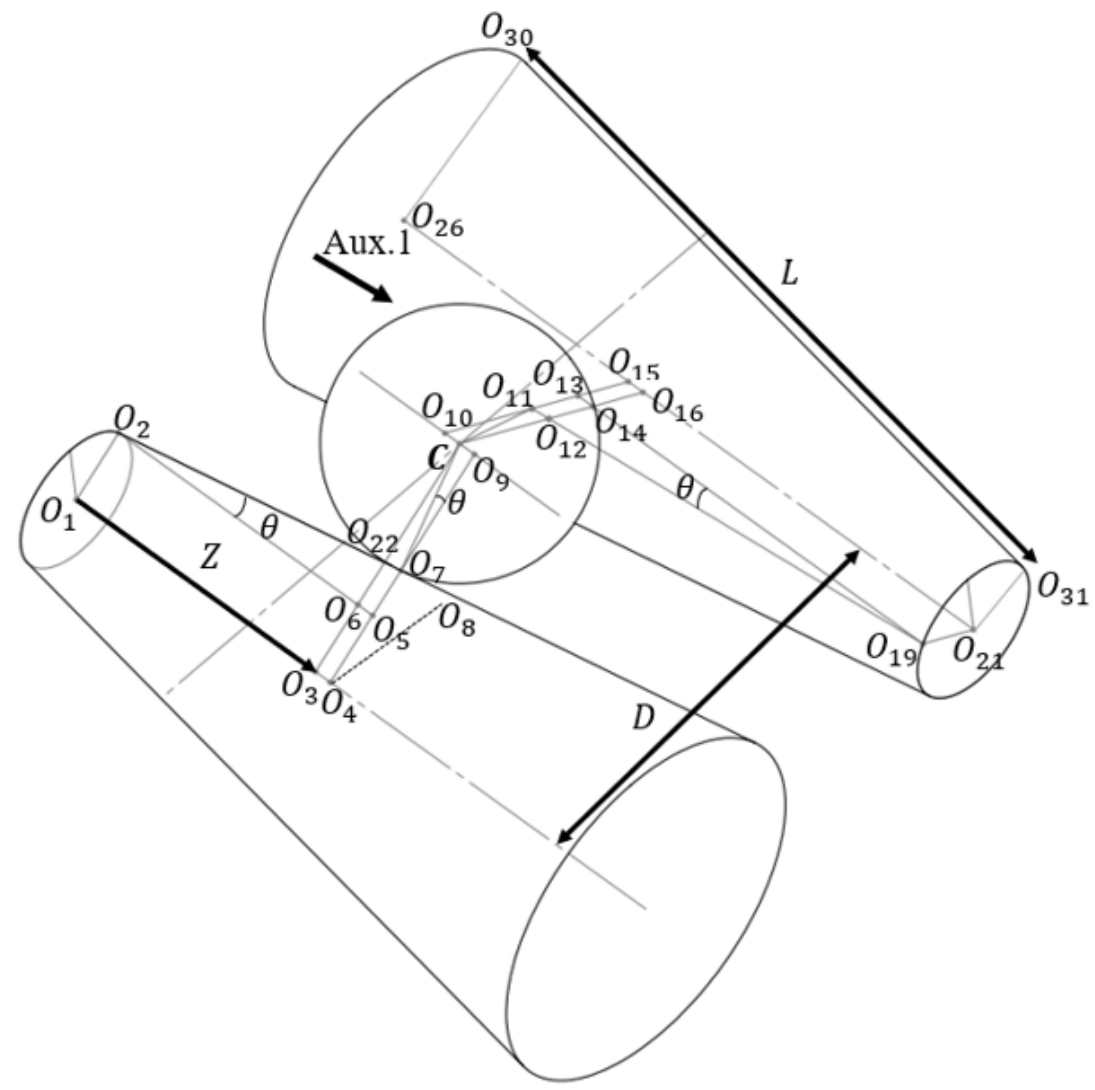

\begin{tabular}{|l|c|}
\hline \multicolumn{2}{|c|}{ First Prototype Dimensions } \\
\hline$R\left(\left|\overrightarrow{\mathrm{CO}_{7}}\right|\right):$ & $17.5 \mathrm{~mm}$ \\
\hline$r_{1}\left(\left|\overrightarrow{\mathrm{O}_{1} \mathrm{O}_{2}}\right|\right):$ & $10 \mathrm{~mm}$ \\
\hline$L\left(\left|\overrightarrow{\mathrm{O}_{30} \mathrm{O}_{31}}\right|\right):$ & $101.12 \mathrm{~mm}$ \\
\hline$\theta:$ & $0.14 \mathrm{rad}$ \\
\hline$D:$ & $59 \mathrm{~mm}$ \\
\hline
\end{tabular}

Figure 10. Isometric view of the drive

In Figure 10, the isometric view of the CVT drive is presented. There is a sphere located above the cones, and all the calculations are carried out by considering this structure. The other sphere, which is located below the cones, is not taken into account since investigating one sphere interaction is sufficient to understand how the system works. Additionally, since the two spheres are located along the same vertical axis, (i.e., they always move symmetrically with respect to the $\mathrm{O}_{1} \mathrm{O}_{3} \mathrm{O}_{16} \mathrm{O}_{26}$ plane), it is sufficient to carry out the calculations with one of the spheres.

The properties of the distance vectors generated with respect to the specific points on Figure 10 are presented below:

$$
\overrightarrow{O_{1} O_{4}}\left\|\overrightarrow{O_{2} O_{5}}\right\| \overrightarrow{O_{10} O_{9}}\left\|\overrightarrow{O_{13} O_{19}}\right\| \overrightarrow{O_{15} O_{21}}
$$




$$
\overrightarrow{\mathrm{CO}_{7}} \perp \overrightarrow{\mathrm{O}_{2} \mathrm{O}_{7}} \text { and } \overrightarrow{\mathrm{CO}_{11}} \perp \overrightarrow{\mathrm{O}_{19} \mathrm{O}_{11}}
$$

The design parameters are identified as $R, r_{1}, L, \theta, D$ where:

$R\left(\left|\overrightarrow{\mathrm{CO}_{7}}\right|\right): \quad$ Radius of the sphere

$r_{1}\left(\left|\overrightarrow{O_{1} O_{2}}\right|\right): \quad$ Minimum cone radius

$L\left(\left|\overrightarrow{O_{30} O_{31}}\right|\right): \quad$ The length of the side of the cone

$\theta$ : $\quad$ Cone angle

$D: \quad$ The distance between the cone's rotation axes

$Z: \quad$ The distance of the center of the sphere from $O_{1}$

The necessary dimensions for the geometrical analysis are defined and calculated in Equations 3.4 to 3.9 .

$$
\begin{gathered}
\left|\overrightarrow{O_{22} O_{7}}\right|=\left|\overrightarrow{C O_{10}}\right|=\left|\overrightarrow{C O_{9}}\right|=\left|\overrightarrow{O_{6} O_{5}}\right|=\left|\overrightarrow{O_{11} O_{12}}\right|=\left|\overrightarrow{O_{13} O_{14}}\right|=\left|\overrightarrow{O_{15} O_{16}}\right|=R \sin \theta \\
\left|\overrightarrow{O_{1} O_{3}}\right|=\left|\overrightarrow{O_{2} O_{6}}\right|=\left|\overrightarrow{O_{26} O_{16}}\right|=Z \\
\left|\overrightarrow{O_{26} O_{21}}\right|=L \cos \theta \\
\left|\overrightarrow{O_{16} O_{21}}\right|=L \cos \theta-Z \\
\left|\overrightarrow{O_{2} O_{5}}\right|=\left|\overrightarrow{O_{2} O_{6}}\right|+\left|\overrightarrow{O_{6} O_{5}}\right|=Z+R \sin \theta \\
\left|\overrightarrow{O_{19} O_{13}}\right|=\left|\overrightarrow{O_{21} O_{15}}\right|=\left|\overrightarrow{O_{16} O_{21}}\right|+\left|\overrightarrow{O_{16} O_{15}}\right|=L \cos \theta-Z+R \sin \theta
\end{gathered}
$$

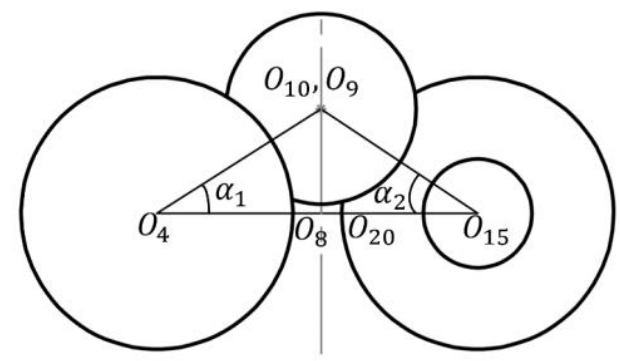

Figure 11. Front view of the drive (Aux.1)

Figure 11 presents the auxiliary view denoted with Aux.1 in Figure 10, which is the view along $\overrightarrow{O_{1} O_{3}}$. Based on Figure 10 and 11, the triangles presented in Figure 12 are formed. 


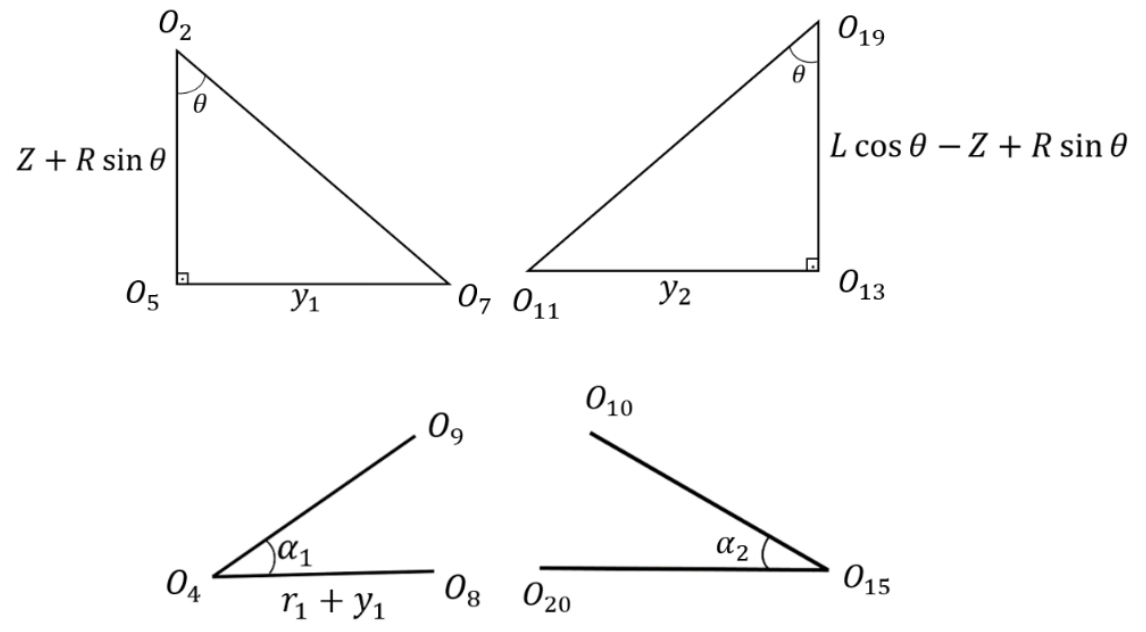

Figure 12. Illustration of $1^{\text {st }}$ group of triangles

Since $\left|\overrightarrow{O_{2} O_{5}}\right|$ and $\left|\overrightarrow{O_{19} O_{13}}\right|$ are known as functions of design parameters, $y_{1}$ and $y_{2}$ parameters presented in Figure 12 are calculated as follows:

$$
\begin{gathered}
y_{1}=\tan \theta(Z+R \sin \theta) \\
y_{2}=\tan \theta(L \cos \theta-Z+R \sin \theta)
\end{gathered}
$$

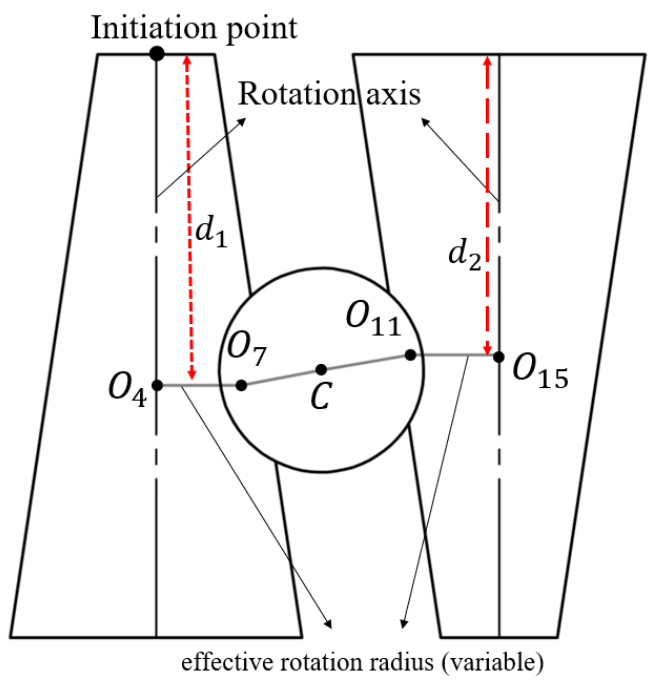

Figure 13. Top view of the drive

In Figure 13, $O_{7}$ and $O_{11}$ are contact points of the sphere with the cone on the left and the cone on the right, respectively. It is clearly observed that these points are not along the same horizontal axis. Hence, effective radii do not have the same distance from the initiation point for any location of the sphere $\left(d_{1} \neq\right.$ $\left.d_{2}\right)$. These radii $\left(r_{2}=\left|\overrightarrow{O_{4} O_{7}}\right|\right.$ and $\left.r_{3}=\left|\overrightarrow{O_{15} O_{11}}\right|\right)$ are calculated in Equations 3.12 and 3.13 by drawing two lines from these contact points to the rotation axes of the respective cones. These drawn lines are perpendicular to the rotation axis of the cones. 


$$
\begin{gathered}
r_{2}=\left|\overrightarrow{O_{4} O_{7}}\right|=y_{1}+r_{1} \\
r_{3}=\left|\overrightarrow{O_{11} O_{15}}\right|=y_{2}+r_{1}
\end{gathered}
$$

Consequently, in practice, the effective radius $r_{2}$ cannot be as small as $r_{1}$ even if the sphere is located at the very top location (near initiation point) of the CVT drive according to the sketch in Figure 13.

Considering Figure 12 and 13, since $\mathrm{O}_{9} \mathrm{O}_{4} \mathrm{O}_{8}$ and $\mathrm{O}_{10} \mathrm{O}_{15} \mathrm{O}_{20}$ define two half triangles, the complete triangle presented in Figure 14 can be formed. This situation can also be observed in Figure 11. Thus, $r_{L}$ and $r_{R}$ lengths are computed based on this triangle in Equation 3.14 and 3.15.

$$
\begin{gathered}
r_{L}=\left|\overrightarrow{O_{9} O_{4}}\right|=r_{1}+y_{1}+R \cos \theta \\
r_{R}=\left|\overrightarrow{O_{10} O_{15}}\right|=r_{1}+y_{2}+R \cos \theta
\end{gathered}
$$

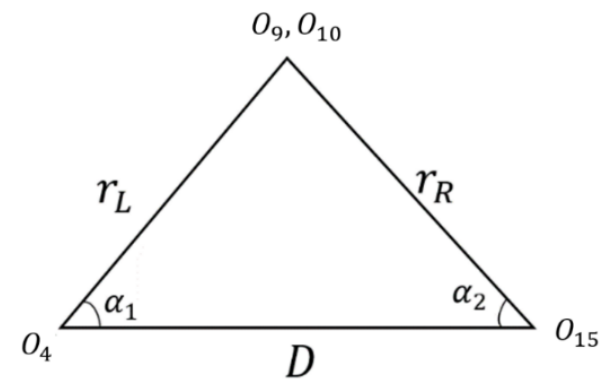

Figure 14. The illustration of $\triangle O_{9} O_{4} O_{15}$

Cosine theorem is implemented in Equation 3.16 and 3.17 to compute $\alpha_{1}$ and $\alpha_{2}$.

$$
\begin{aligned}
& \cos \alpha_{1}=\left(r_{L}^{2}+D^{2}-r_{R}^{2}\right) /\left(2 r_{L} D\right) \\
& \cos \alpha_{2}=\left(r_{R}^{2}+D^{2}-r_{L}^{2}\right) /\left(2 r_{R} D\right)
\end{aligned}
$$

Since $\left|\overrightarrow{\mathrm{CO}_{7}}\right|,\left|\overrightarrow{\mathrm{O}_{2} \mathrm{O}_{6}}\right|,\left|\overrightarrow{\mathrm{O}_{19} \mathrm{O}_{14}}\right|$ and $\left|\overrightarrow{\mathrm{CO}_{11}}\right|$ are known, $\left|\overrightarrow{\mathrm{CO}_{22}}\right|,\left|\overrightarrow{\mathrm{O}_{22} \mathrm{O}_{6}}\right|,\left|\overrightarrow{\mathrm{O}_{12} \mathrm{O}_{14}}\right|$ and $\left|\overrightarrow{\mathrm{CO}_{12}}\right|$ are calculated from trigonometric relations indicated in Figure 15.

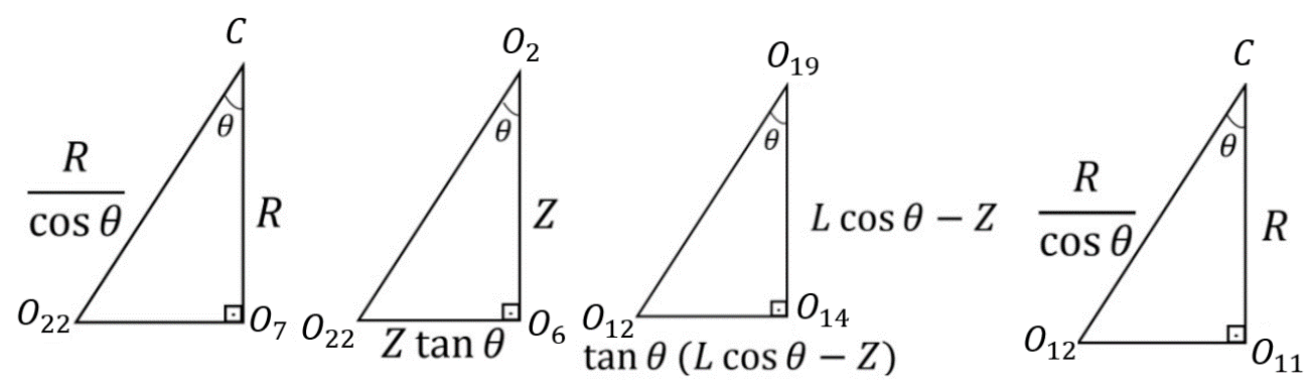

Figure 15. Illustration of $2^{\text {nd }}$ group of triangles

Also, by using these triangles, the following dimensions are obtained from Figure 16:

$$
\begin{gathered}
r_{C L}=\left|\overrightarrow{C O_{3}}\right|=r_{1}+Z \tan \theta+(R / \cos \theta) \\
r_{C R}=\left|\overrightarrow{C O_{16}}\right|=r_{1}+\tan \theta(L \cos \theta-Z)+(R / \cos \theta)
\end{gathered}
$$




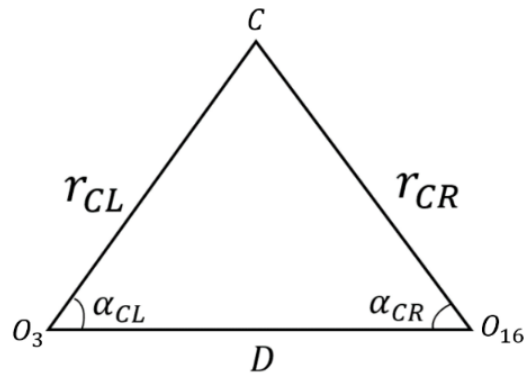

Figure.16 The illustration of the center of the sphere with the help of $\triangle \mathrm{CO}_{3} \boldsymbol{O}_{16}$

Based on Figure 16, using the cosine theorem, $\alpha_{C L}$ and $\alpha_{C R}$ are computed as follows:

$$
\begin{aligned}
& \cos \alpha_{C L}=\left(r_{C L}^{2}+D^{2}-r_{C R}^{2}\right) /\left(2 r_{C L} D\right) \\
& \cos \alpha_{C R}=\left(r_{C R}^{2}+D^{2}-r_{C L}^{2}\right) /\left(2 r_{C R} D\right)
\end{aligned}
$$

Obviously, $\alpha_{1}=\alpha_{C L}$ and $\alpha_{2}=\alpha_{C R}$. The position vector of the center of the sphere with respect to $0^{\text {th }}$ frame, $\vec{P}_{C}\left(\overrightarrow{O_{1} C}\right)$, is computed in Equation 3.22 based on the sketch presented in Figure 17.

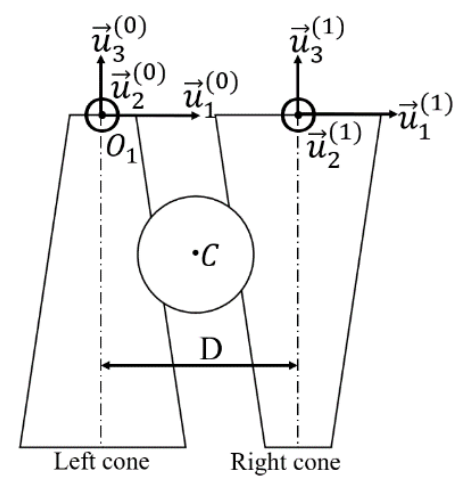

Figure 17. The illustration of the $0^{\text {th }}$ and $1^{\text {st }}$ frame

$$
\vec{P}_{C}=\overrightarrow{O_{1} C}=\left(r_{C L} \cos \alpha_{C L}\right) \vec{u}_{1}^{(0)}+\left(r_{C L} \sin \alpha_{C L}\right) \vec{u}_{2}^{(0)}+Z \vec{u}_{3}^{(0)}
$$

In Figure 18, the translation of the center of the sphere, as it is moved from one end of the drive to the other end, is presented. In order to clearly visualize this motion, the path of the sphere's center on the $\vec{u}_{1}^{(0)}-\vec{u}_{2}^{(0)}(\mathrm{X}-\mathrm{Y})$ plane, on the $\vec{u}_{1}^{(0)}-\vec{u}_{3}^{(0)}(\mathrm{X}-\mathrm{Z})$ plane, and in the Cartesian frame are illustrated in this figure. These three graphs are drawn by making use of Equation 3.22. 


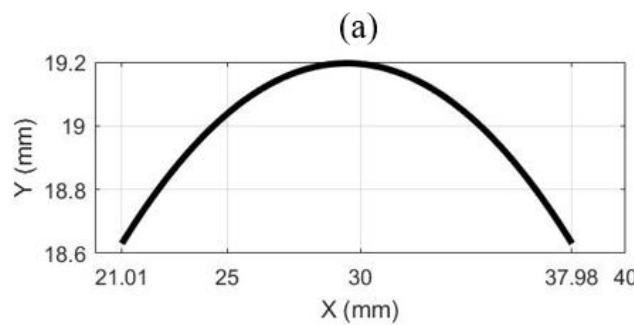

(b)

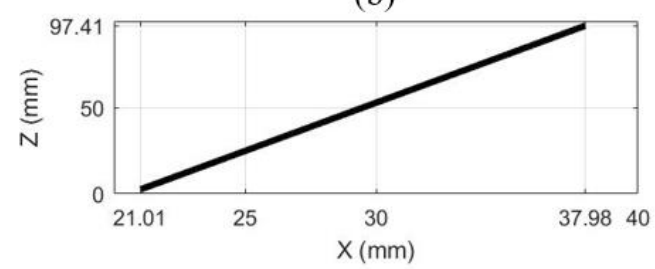

(c)

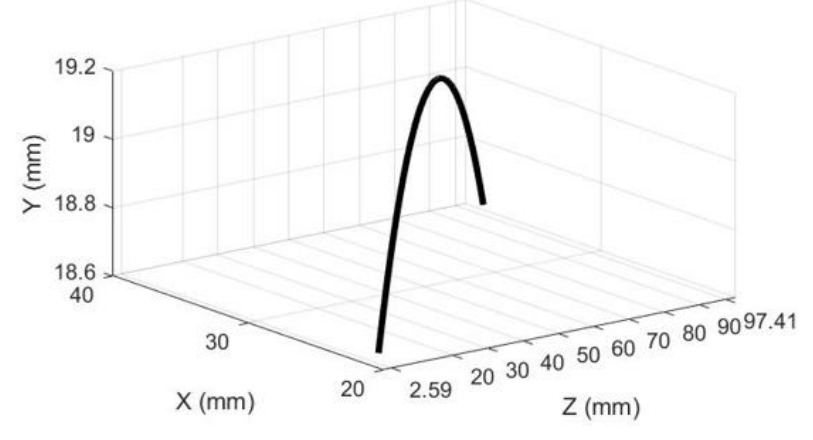

Figure 18. The projection of the position variation of the center of the sphere

(a) $\mathrm{X}-\mathrm{Y}$ plane, (b) X-Z plane, (c) isometric view

Subsequently, the position vector of the left cone contact point with respect to $0^{\text {th }}$ frame is calculated. The left cone contact point is $O_{7}$ as shown in Figure 10 and 13. Therefore, $\vec{P}_{L}$ is defined as $\overrightarrow{O_{1} O_{7}}$ (Figure 10 ) and its vector equation is presented in Equation 3.23. Note that $r_{2}$ represents the $\left|\overrightarrow{O_{4} O_{7}}\right|$ dimension indicated in Equation 3.12.

$$
\vec{P}_{L}=\left(r_{2} \cos \alpha_{1}\right) \vec{u}_{1}^{(0)}+\left(r_{2} \sin \alpha_{1}\right) \vec{u}_{2}^{(0)}+(Z+R \sin \theta) \vec{u}_{3}^{(0)}
$$

The position vector of the right cone contact point with respect to the $1^{\text {st }}$ frame is written with the help of $\overrightarrow{O_{26} O_{11}}$ since the right cone contact point is $O_{11}$ (Figure 10). Therefore, the vector formula for $\overrightarrow{O_{26} O_{11}}$ is stated in Equation 3.24, and the sign of $\vec{u}_{1}^{(1)}$ component is negative according to the definition of the $1^{\text {st }}$ frame presented in Figure 17. Note that $r_{3}$ represents the $\left|\overrightarrow{O_{11} O_{15}}\right|$ dimension indicated in Equation 3.13 .

$$
\vec{P}_{R}=\left(-r_{3} \cos \alpha_{2}\right) \vec{u}_{1}^{(1)}+\left(r_{3} \sin \alpha_{2}\right) \vec{u}_{2}^{(1)}+(Z-R \sin \theta) \vec{u}_{3}^{(1)}
$$

In order to represent $\vec{P}_{R}$ vector in $0^{\text {th }}$ frame, the homogenous transformation matrix $\widehat{H}^{(0,1)}$ is defined in Equation 3.25, and the necessary transformations are performed in Equation 3.26 and 3.27.

$$
\begin{gathered}
\widehat{H}^{(0,1)}=\left[\begin{array}{cccc}
1 & 0 & 0 & D \\
0 & 1 & 0 & 0 \\
0 & 0 & 1 & 0 \\
0 & 0 & 0 & 1
\end{array}\right] \\
\bar{P}_{R}^{*(0)}=\widehat{H}^{(0,1)} \bar{P}_{R}^{*(1)}, \text { where } \bar{P}_{R}^{*(i)}=\left[\begin{array}{c}
\bar{P}_{R}^{(i)} \\
1
\end{array}\right] \\
\vec{P}_{R}=\left(-r_{3} \cos \alpha_{2}+D\right) \vec{u}_{1}^{(0)}+\left(r_{3} \sin \alpha_{2}\right) \vec{u}_{2}^{(0)}+(Z-R \sin \theta) \vec{u}_{3}^{(0)}
\end{gathered}
$$

After determining the position vector of the contact points with respect to the inertial reference frame ( $0^{\text {th }}$ frame), the boundary conditions of the contact points in terms of the $Z$ parameter are determined. Considering Figures 10 and 13, it is clear that the minimum $Z$ value measured from the inertial reference frame is equal to the $\vec{u}_{3}^{(0)}$ component of $\vec{P}_{R}$ (Equation 3.28). In other words, this component is set equal to zero (Equation 3.29) so that the minimum $Z$ value can be computed (Equation 3.30). The reason for this is that the position of the right cone contact point is always closer to the inertial reference frame with respect to the left cone contact point, which can be observed in Figure 13. 


$$
\begin{gathered}
\vec{P}_{R}=\left(-r_{3} \cos \alpha_{2}\right) \vec{u}_{1}^{(1)}+\left(r_{3} \sin \alpha_{2}\right) \vec{u}_{2}^{(1)}+(Z-R \sin \theta) \vec{u}_{3}^{(1)} \\
0=Z_{\text {min }}-R \sin \theta \\
Z_{\text {min }}=R \sin \theta
\end{gathered}
$$

On the other hand, the maximum $Z$ value is obtained from the $\vec{u}_{3}^{(0)}$ element of $\vec{P}_{L}$ presented in Equation 3.23. Therefore, this component of Equation 3.23 is set equal to $L \cos \theta$, which is the maximum distance from the inertial reference frame according to Figure 10. The necessary calculations are performed in Equation 3.31 and 3.32.

$$
\begin{aligned}
& Z_{\text {max }}+R \sin \theta=L \cos \theta \\
& Z_{\text {max }}=L \cos \theta-R \sin \theta
\end{aligned}
$$

In this way, the boundary conditions of the $Z$ values are computed. These boundaries help us to determine the maximum and minimum transmission ratios achievable by the CVT drive since this ratio is varied due to the location of the contact points with respect to the inertial reference frame.

Up to this point, the location of the sphere has been calculated with respect to the inertial reference frame as a function of design parameters. However, the orientation change of the sphere has not yet been investigated. Here, the investigation of this issue is addressed. In Figure 19, the side view of the CVT drive is presented. A plane is defined by making use of $C, O_{7}$ and $O_{11}$ points that were presented in Figure 10. The angle between this plane and the $\vec{u}_{1}^{(0)}-\vec{u}_{2}^{(0)}$ plane is identified as $\gamma$ in Figure 19.

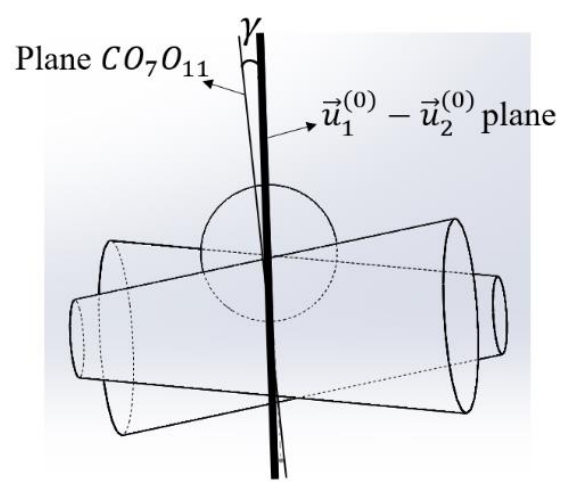

Figure 19. The illustration of the $\gamma$ angle

In Figure 20, which is a zoomed view of Figure 10, there are three frames identified with numbers 2 , 3 and 4. The second frame has the same orientation as the inertial reference frame and the first frame, as presented in Figure 17. Therefore, rotations with respect to the second frame can be considered as the rotations with respect to the inertial reference frame. In the third frame, it is aimed to determine the axis which is tangent to the left cone contact point along the longitudinal direction $\left(\vec{u}_{3}^{(3)}\right)$. Thus, the rotation $\left(\theta_{11}\right)$ is performed around the $\vec{u}_{2}^{(2)}$ axis as can be seen in Figure 20 and 24. Afterward, the axes that are aligned with the left cone contact point are specified by rotating the third frame around $\vec{u}_{3}^{(3)}$ due to the fact that $\vec{u}_{3}^{(3)}$ is tangent to the sphere and the left cone contact point. Thus, the investigated axis, which describes the surface normal of the left cone contact point, is assigned as $\vec{u}_{1}^{(4)}$, which is aligned with $\overrightarrow{O_{23} C}$. Furthermore, $\vec{u}_{2}^{(4)}$ defines the direction of the tangential velocity and the forces acting between the cones and the sphere during the transmission of power from one cone to the other. 


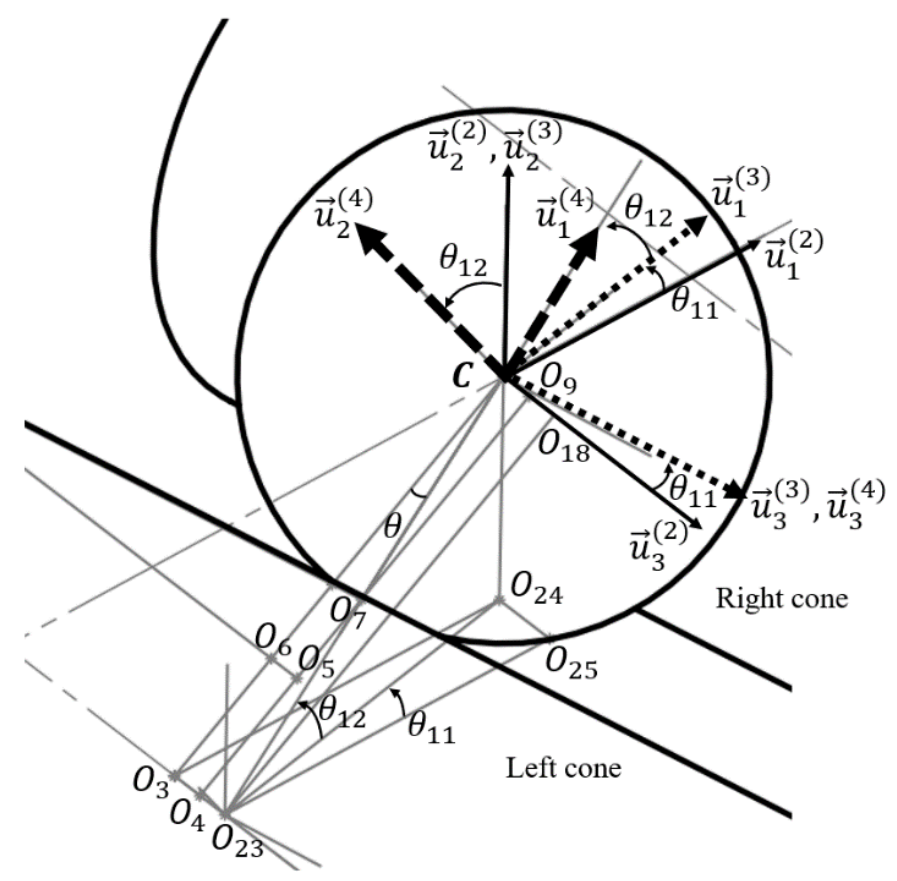

Figure 20. Detailed view of the contact point between the left cone and the sphere

After identifying the required angles, the parametric equalities of these angles are investigated with the help of the CAD model. First, $\triangle \mathrm{CO}_{3} \mathrm{O}_{23}$, which is presented as the triangle on the left in Figure 21, is evaluated. In this case, $\overrightarrow{\mathrm{CO}_{3}}$ is perpendicular to $\vec{u}_{2}^{(0)}=\vec{u}_{2}^{(2)}$, which is the rotation axis of the input cone. Since $\left|\overrightarrow{\mathrm{CO}_{3}}\right|$ is already defined as $r_{C L}$, and the angle between the $\left|\overrightarrow{\mathrm{CO}_{3}}\right|$ and $\left|\overrightarrow{\mathrm{CO}_{23}}\right|$ is $\theta,\left|\overrightarrow{\mathrm{O}_{3} \mathrm{O}_{23}}\right|$ can be calculated. Afterwards, $\left|\overrightarrow{\mathrm{O}_{3} \mathrm{O}_{24}}\right|$ and $\left|\overrightarrow{\mathrm{CO}_{24}}\right|$ are determined from $\triangle \mathrm{CO}_{3} \mathrm{O}_{24}$, as shown in detail in Figure 21.

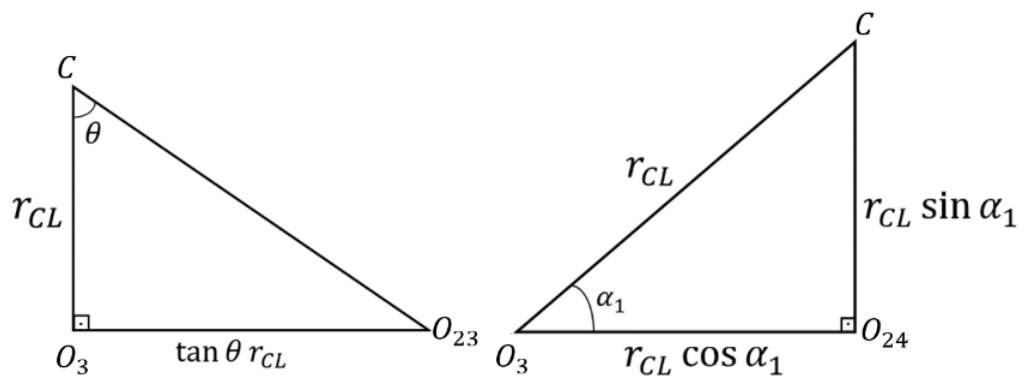

Figure 21. $3^{\text {rd }}$ group of the triangles

To determine the $\theta_{11}, \Delta \mathrm{O}_{23} \mathrm{O}_{3} \mathrm{O}_{24}$ presented in Figure 22 is studied. In this case, it is clear that $\left|\overrightarrow{\mathrm{O}_{3} \mathrm{O}_{24}}\right|$ is parallel to $\left|\overrightarrow{\mathrm{O}_{23} \mathrm{O}_{25}}\right|$, and $\left|\overrightarrow{\mathrm{O}_{3} \mathrm{O}_{23}}\right|$ is parallel to $\left|\overrightarrow{\mathrm{O}_{24} \mathrm{O}_{25}}\right|$. Next, since these terms are computed already, $\theta_{11}$ is obtained based on the illustration in Figure 22. The calculations for $\theta_{11}$ are presented in Equations 3.33 to 3.35 . 


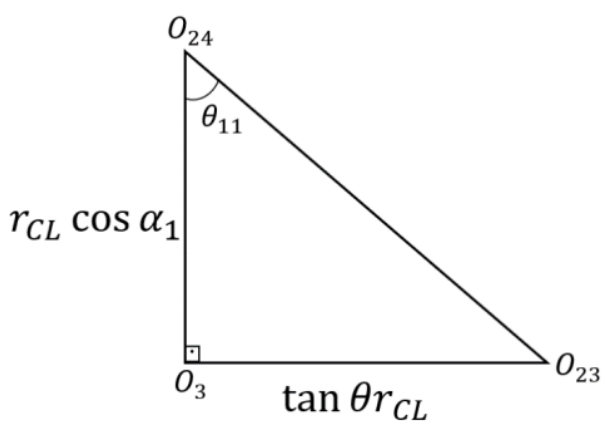

Figure 22. The illustration of the $\Delta \mathrm{O}_{24} \mathrm{O}_{3} \mathrm{O}_{23}$

$$
\begin{gathered}
\left|\overrightarrow{O_{23} O_{24}}\right|=\sqrt{\left(r_{C L} \cos \alpha_{1}\right)^{2}+\left(\tan \theta r_{C L}\right)^{2}} \\
\tan \theta_{11}=\left(\tan \theta r_{C L}\right) /\left(r_{C L} \cos \alpha_{1}\right) \\
\theta_{11}=\tan ^{-1}\left(\left(\tan \theta r_{C L}\right) /\left(r_{C L} \cos \alpha_{1}\right)\right)
\end{gathered}
$$

Finally, $\theta_{12}$ is calculated from $\triangle C O_{24} O_{23}$, which is presented in Figure 23. The necessary mathematical operations for this calculation are shown in Equation 3.36 and 3.37.

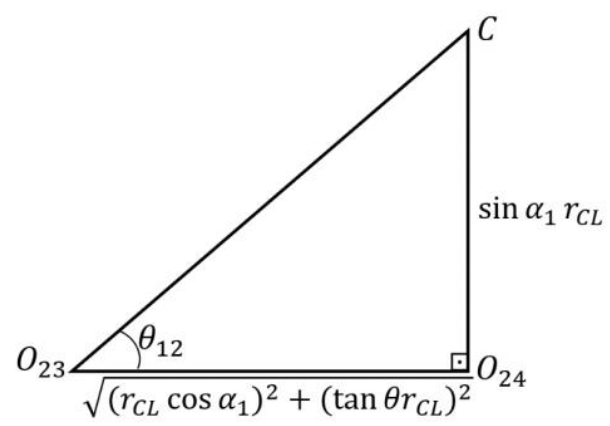

Figure 23. The illustration of the $\Delta \mathrm{CO}_{24} \mathrm{O}_{23}$

$$
\begin{gathered}
\tan \theta_{12}=\left(\sin \alpha_{1} r_{C L}\right) /\left(\sqrt{\left(r_{C L} \cos \alpha_{1}\right)^{2}+\left(\tan \theta r_{C L}\right)^{2}}\right) \\
\theta_{12}=\tan ^{-1}\left(\left(\sin \alpha_{1} r_{C L}\right) /\left(\sqrt{\left(r_{C L} \cos \alpha_{1}\right)^{2}+\left(\tan \theta r_{C L}\right)^{2}}\right)\right)
\end{gathered}
$$

The rotations between the frames are illustrated in Figure 24. Since the orientations of the inertial reference frame and the second frame are the same, the rotation matrix is defined between the second and fourth frames, and its derivation is presented in Equations 3.38 to 3.40 . 

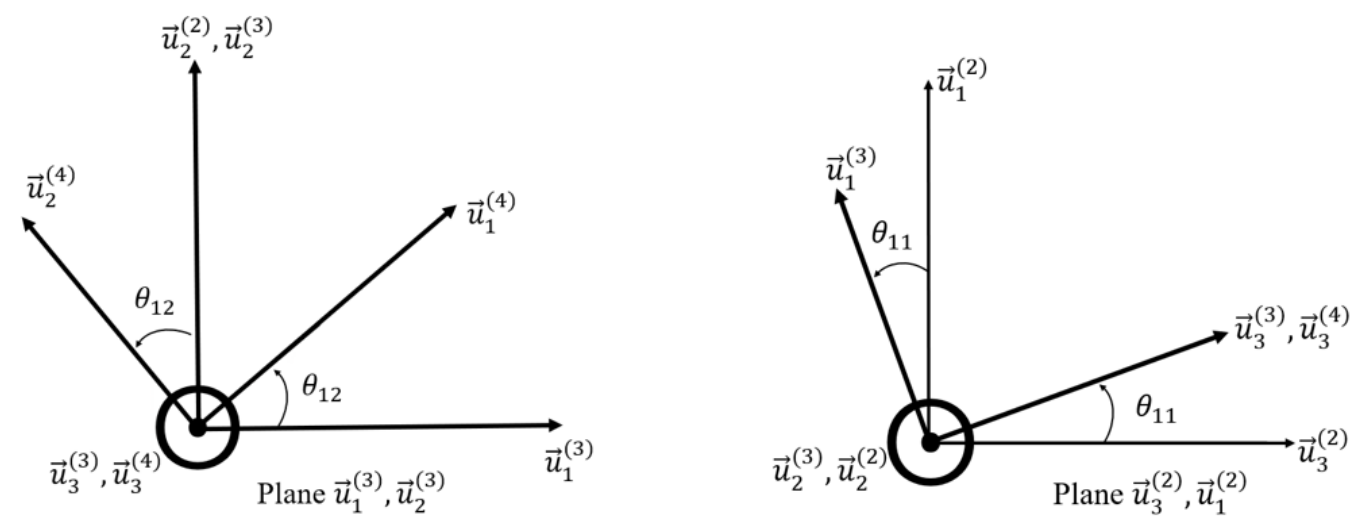

Figure 24. Rotations taking place on the sphere for left contact point

$$
\begin{gathered}
\hat{C}^{(2,4)}=\hat{C}^{(2,3)} \hat{C}^{(3,4)}=e^{\widetilde{u}_{2} \theta_{11}} e^{\widetilde{u}_{3} \theta_{12}} \\
\hat{C}^{(2,3)}=\left[\begin{array}{ccc}
\cos \theta_{11} & 0 & \sin \theta_{11} \\
0 & 1 & 0 \\
-\sin \theta_{11} & 0 & \cos \theta_{11}
\end{array}\right] \\
\hat{C}^{(3,4)}=\left[\begin{array}{ccc}
\cos \theta_{12} & -\sin \theta_{12} & 0 \\
\sin \theta_{12} & \cos \theta_{12} & 0 \\
0 & 0 & 1
\end{array}\right] \\
\hat{C}^{(2,4)}=\left[\begin{array}{ccc}
\cos \theta_{11} \cos \theta_{12} & -\cos \theta_{11} \sin \theta_{12} & \sin \theta_{11} \\
\sin \theta_{12} & \cos \theta_{12} & 0 \\
-\cos \theta_{12} \sin \theta_{11} & \sin \theta_{11} \sin \theta_{12} & \cos \theta_{11}
\end{array}\right]
\end{gathered}
$$

where $\hat{C}^{(i, j)}$ is the transformation matrix used as the rotation matrix between the $i^{\text {th }}$ and $j^{\text {th }}$ frame. The open form of the rotation matrix in Equation 3.41, which is a function of design parameters $\left(R, r_{1}, L, D\right.$, $\theta$ ), is not presented here since the computations for this matrix's elements are already introduced in this section.

In the next step, the rotations between the sphere and the right cone contact point are analyzed. Since the surface normal for the right contact point $\left(\overrightarrow{\mathrm{CO}_{11}}\right)$ and the left contact point $\left(\overrightarrow{\mathrm{CO}_{7}}\right)$ do not have the same orientation with respect to the inertial reference frame, each of them is considered separately. The orientation of the surface normal for the right contact point with respect to the inertial reference frame is indicated in Figure 25. 


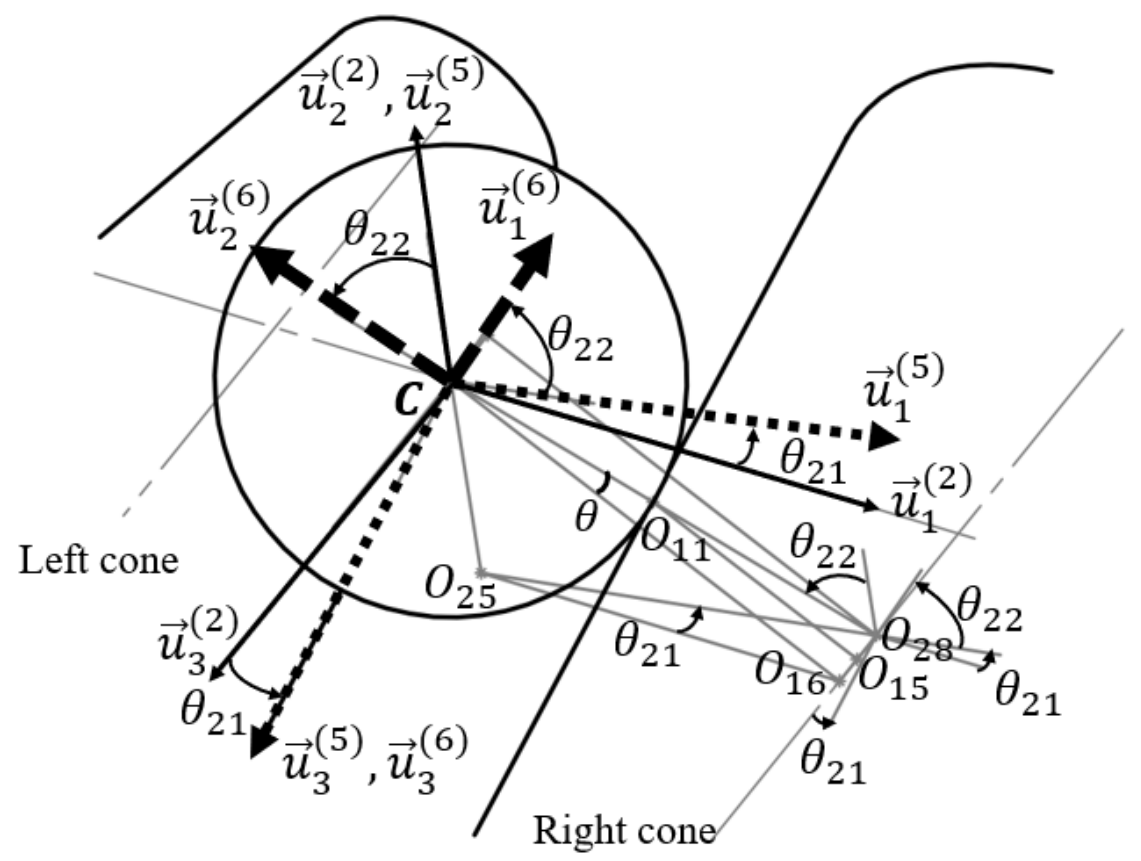

Figure 25. Detailed view of the contact point between the right contact point and the sphere

In Figure 25, the second frame is the common frame for the two contact points, which is also indicated in Figure 20. Hence, the connection between these calculations is achieved with the help of this frame. Similar to the previous investigation, there are three frames denoted as second, fifth and sixth frames in this investigation. Thus, in the fifth frame, the axes that are tangent to the sphere along the longitudinal direction $\left(\vec{u}_{3}^{(5)}\right)$ are obtained. Finally, in the sixth frame, the axis $\overrightarrow{O_{28} C}$, which is aligned with the contact point of the right cone and the sphere, is acquired and defined as $\vec{u}_{2}^{(6)}$.

Afterward, the parametric calculations are performed with the help of the triangles presented in Figure 26. First, $\triangle \mathrm{CO}_{16} \mathrm{O}_{28}$ is investigated and $\left|\overrightarrow{\mathrm{O}_{16} \mathrm{O}_{28}}\right|$ is found from the trigonometric relations. Then, $\left|\overrightarrow{\mathrm{CO}_{25}}\right|$ and $\left|\overrightarrow{O_{25} O_{16}}\right|$ are obtained, as presented on the right triangle in Figure 26.
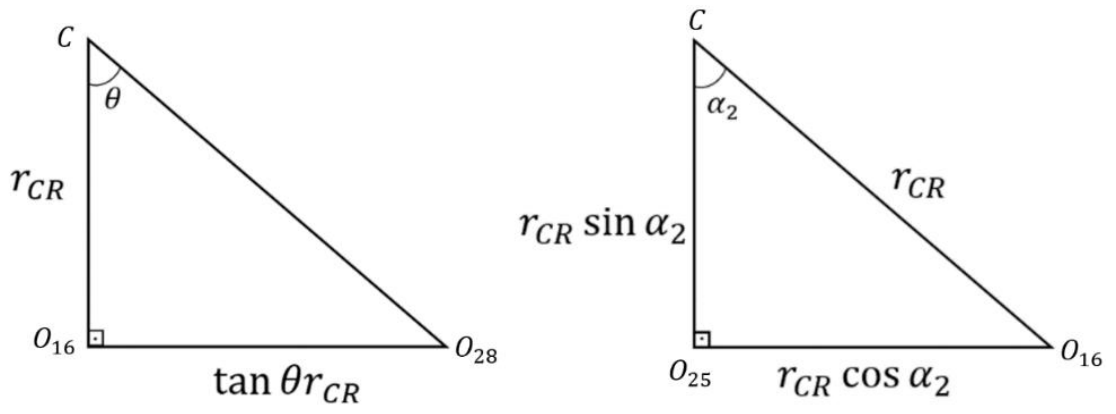

Figure 26. $4^{\text {th }}$ group of triangles

Consequently, $\theta_{21}$ is calculated from $\Delta \mathrm{O}_{25} \mathrm{O}_{16} \mathrm{O}_{28}$, as presented in Figure 27. In order to accomplish this, $\left|\overrightarrow{\mathrm{O}_{25} \mathrm{O}_{28}}\right|$ is calculated as shown in Equation 3.42, and then, $\theta_{21}$ is acquired from the trigonometric relations presented in Equation 3.43 and 3.44 . 


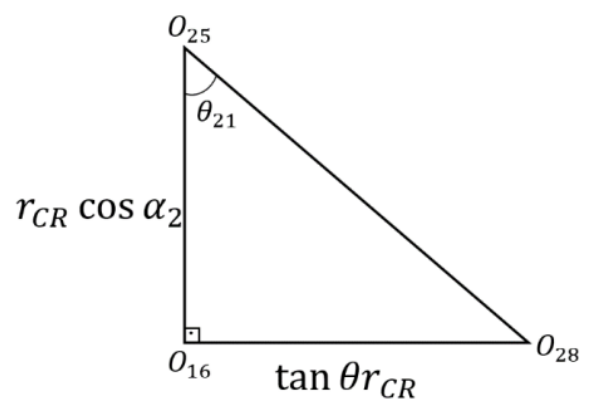

Figure 27. The illustration of $\Delta \mathrm{O}_{25} \mathrm{O}_{16} \mathrm{O}_{28}$

$$
\begin{gathered}
\left|\overrightarrow{O_{25} O_{28}}\right|=\sqrt{\left(r_{C R} \cos \alpha_{2}\right)^{2}+\left(\tan \theta r_{C R}\right)^{2}} \\
\tan \theta_{21}=\left(\tan \theta r_{C R}\right) /\left(r_{C R} \cos \alpha_{2}\right) \\
\theta_{21}=\tan ^{-1}\left(\left(\tan \theta r_{C R}\right) /\left(r_{C R} \cos \alpha_{2}\right)\right)
\end{gathered}
$$

As the first step in obtaining $\theta_{22}, \theta_{23}$ is computed as shown in Equations 3.45 and 3.46 based on $\triangle \mathrm{CO}_{25} \mathrm{O}_{28}$ illustrated in Figure 28. Finally, $\theta_{22}$ is computed as presented in Equation 3.47.

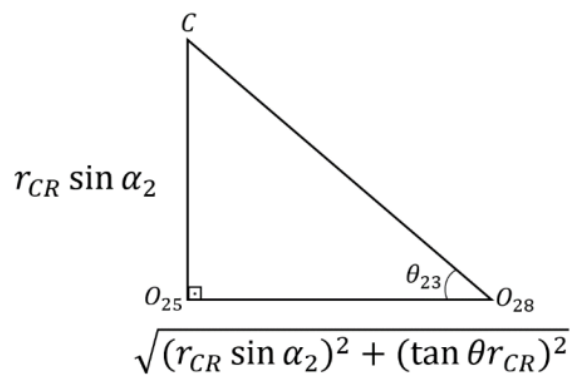

Figure 28. The illustration of the $\Delta \mathrm{CO}_{25} \mathrm{O}_{28}$

$$
\begin{gathered}
\tan \theta_{23}=\left(r_{C R} \sin \alpha_{2}\right) /\left(\sqrt{\left(r_{C R} \sin \alpha_{2}\right)^{2}+\left(\tan \theta r_{C R}\right)^{2}}\right) \\
\theta_{23}=\tan ^{-1}\left(\left(r_{C R} \sin \alpha_{2}\right) /\left(\sqrt{\left(r_{C R} \sin \alpha_{2}\right)^{2}+\left(\tan \theta r_{C R}\right)^{2}}\right)\right) \\
\theta_{22}=90-\theta_{23}
\end{gathered}
$$

The rotations defined on the sphere for the right contact point in between the frames are illustrated in Figure 29. 

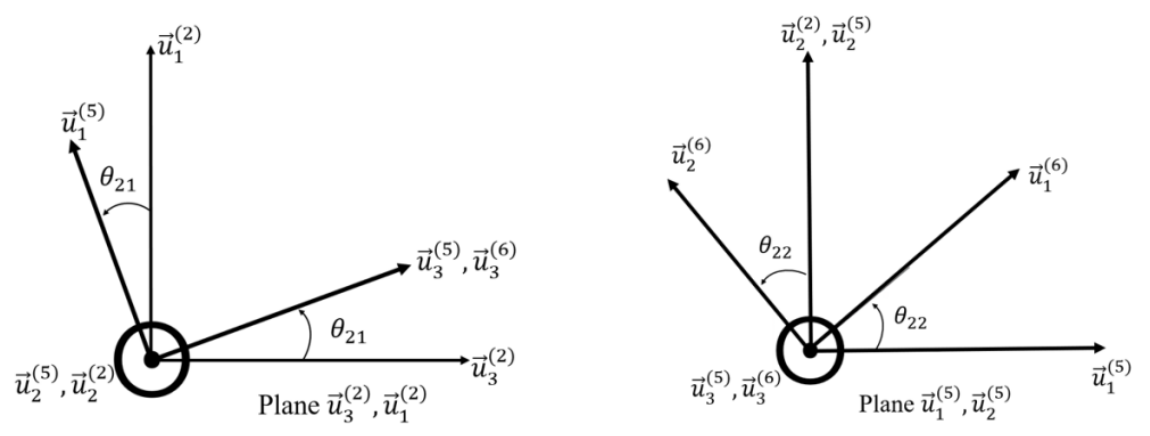

Figure 29. Rotations taking place on the sphere for right contact point

Since the orientations of the inertial reference frame and the second frame are the same, the calculation of the rotation matrix between the second and sixth frames are carried out in Equations 3.48 to 3.50.

$$
\begin{gathered}
\hat{C}^{(2,6)}=\hat{C}^{(2,5)} \hat{C}^{(5,6)}=e^{\widetilde{u}_{2} \theta_{11}} e^{\widetilde{u}_{3} \theta_{12}} \\
\hat{C}^{(2,5)}=\left[\begin{array}{ccc}
\cos \theta_{21} & 0 & \sin \theta_{21} \\
0 & 1 & 0 \\
-\sin \theta_{21} & 0 & \cos \theta_{21}
\end{array}\right] \\
\hat{C}^{(5,6)}=\left[\begin{array}{ccc}
\cos \theta_{22} & -\sin \theta_{22} & 0 \\
\sin \theta_{22} & \cos \theta_{22} & 0 \\
0 & 0 & 1
\end{array}\right] \\
\hat{C}^{(2,6)}=\left[\begin{array}{ccc}
\cos \theta_{21} \cos \theta_{22} & -\cos \theta_{21} \sin \theta_{22} & \sin \theta_{21} \\
\sin \theta_{22} & \cos \theta_{22} & 0 \\
-\cos \theta_{22} \sin \theta_{21} & \sin \theta_{21} \sin \theta_{22} & \cos \theta_{21}
\end{array}\right]
\end{gathered}
$$

The open form of the rotation matrix presented in Equation 3.51 as a function of design parameters $\left(R, r_{1}, L, D, \theta\right)$ is not presented here since the computations for this matrix's elements are already introduced in this section.

In Figure 30, the largest cone radius $r_{12}$ is presented, and its relation to the minimum radius $r_{1}$ is defined in Equation 3.52.

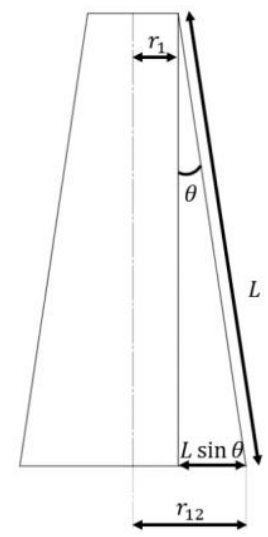

Figure 30. The geometric illustration of the largest cone radius

$$
r_{12}=r_{1}+l \sin \theta
$$




\section{Verification of the Geometrical Analysis}

In this section, the validation for the calculation of the sphere's orientation in terms of design parameters are provided. The angles that are calculated in this section are measured for different $Z$ values from the Solidworks model of this CVT drive system. The measurement accuracy is assigned to be $0.000001 \mathrm{~mm}$ in Solidworks software. These angles defining the geometry are also calculated by using the formulations given in this section by using a Matlab m-file code. The results of the Solidworks measurements and Matlab calculations are reported in Table 1 along with the differences between them as error percentage.

Table 1 The comparison between the measured and calculated angles

\begin{tabular}{|c|c|c|c|c|c|c|c|c|c|c|c|c|}
\hline & \multicolumn{3}{|c|}{$\theta_{11}$} & \multicolumn{3}{|c|}{$\theta_{12}$} & \multicolumn{3}{|c|}{$\theta_{21}$} & \multicolumn{3}{|c|}{$\boldsymbol{\theta}_{22}$} \\
\hline$Z$ & 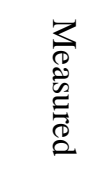 & 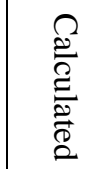 & $\begin{array}{l}\frac{T}{9} \\
\stackrel{9}{9} \\
d^{9}\end{array}$ & $\begin{array}{l}3 \\
\mathbb{8} \\
0 \\
0 \\
0 \\
8\end{array}$ & 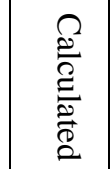 & $\begin{array}{l}\text { T) } \\
\stackrel{9}{9} \\
0\end{array}$ & $\begin{array}{l}3 \\
3 \\
8 \\
0 \\
0 \\
8 \\
2\end{array}$ & 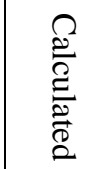 & 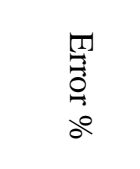 & 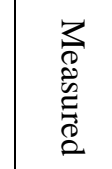 & 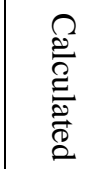 & $\begin{array}{l}\frac{\pi}{3} \\
\stackrel{0}{0} \\
09\end{array}$ \\
\hline 3 & $11.31^{\circ}$ & $11.31^{\circ}$ & $0.002^{\circ}$ & $40.91^{\circ}$ & $40.91^{\circ}$ & $0.0001^{\circ}$ & $9.48^{\circ}$ & $9.48^{\circ}$ & $0.0003^{\circ}$ & $64.12^{\circ}$ & $64.12^{\circ}$ & $0.0003^{\circ}$ \\
\hline 12 & $11.02^{\circ}$ & $11.02^{\circ}$ & $0.0002^{\circ}$ & $39.15^{\circ}$ & $39.15^{\circ}$ & $0.0001^{\circ}$ & $9.59^{\circ}$ & $9.59^{\circ}$ & $0.0003^{\circ}$ & $62.90^{\circ}$ & $62.90^{\circ}$ & $0.0004^{\circ}$ \\
\hline 21 & $10.77^{\circ}$ & $10.77^{\circ}$ & $0.0002^{\circ}$ & $37.49^{\circ}$ & $37.49^{\circ}$ & $0.0007^{\circ}$ & $9.70^{\circ}$ & $9.70^{\circ}$ & $0.0003^{\circ}$ & $61.65^{\circ}$ & $61.65^{\circ}$ & $0.0005^{\circ}$ \\
\hline 62 & $9.94^{\circ}$ & $9.94^{\circ}$ & $0.0003^{\circ}$ & $30.8^{\circ}$ & $30.8^{\circ}$ & $0.0001^{\circ}$ & $10.37^{\circ}$ & $10.37^{\circ}$ & $0.0004^{\circ}$ & $55.43^{\circ}$ & $55.43^{\circ}$ & $0.0001^{\circ}$ \\
\hline 70 & $9.82^{\circ}$ & $9.82^{\circ}$ & $0.0003^{\circ}$ & $29.63^{\circ}$ & $29.63^{\circ}$ & $0.0001^{\circ}$ & $10.55^{\circ}$ & $10.55^{\circ}$ & $0.0004^{\circ}$ & $54.08^{\circ}$ & $54.08^{\circ}$ & $0.0001^{\circ}$ \\
\hline 97 & $9.48^{\circ}$ & $9.48^{\circ}$ & $0.0003^{\circ}$ & $25.87^{\circ}$ & $25.87^{\circ}$ & $0.0008^{\circ}$ & $11.31^{\circ}$ & $11.31^{\circ}$ & $0.0004^{\circ}$ & $49.08^{\circ}$ & $49.08^{\circ}$ & $0.0001^{\circ}$ \\
\hline
\end{tabular}

According to Table 1, the overall average error is calculated as $0.0009 \%$, which means that the differences between the measurements and the calculations are in in the level of numerical error ranges.

\section{Conclusions}

In this paper, a novel variable stiffness joint design based on two-cone friction-drive CVT is introduced. The main reasons that the two-cone friction drive CVT systems are not used in the design of VSA systems are investigated. These reasons are identified as the bidirectional torque transmission problem and position change during the transmission ratio variation problem. To overcome these problems, a significant modification is issued in the two-cone friction-drive CVT design by changing the transmission element to a double sphere. A previously built and reported (Mobedi and Dede, 2018) first prototype, which is used in the proof of concept tests, showed that this new CVT can perform the bidirectional torque transmission.

Nevertheless, the inertia of the cones and the spherical transmission elements produced for the first prototype should be decreased to minimize the minimum impedance displayed to human. Moreover, optimization of the mechanism is important to enhance the application of the area of this new CVT in human-robot interfaces. To implement the optimization, a geometrical analysis is required to identify the capabilities of the device with respect to the design parameters. In this article, geometrical analysis of this new CVT drive is presented, and parametric formulas for the required angles that define the geometry of this drive are derived. These formulas are validated against a Solidworks model of the drive system.

Future studies of this work include static force analysis in order to carry out the optimization of this drive for a haptic device, production of the optimized drive, and finally, design validation tests to measure the performance of the design by using metrics defined for haptic systems. 


\section{Acknowledgments}

This work is supported by The Scientific and Technological Research Council of Turkey via grant number 117M405.

\section{References}

Alò, R., Bottiglione, F., and Mantriota G. (2018). Flywheel-infinitely Variable Transmissions for Energy Recovery Capabilities in Artificial Knee Joints, Mechanics Based Design of Structures and Machines An International Journal, Vol.46, No.3, pp. 333-46.

Carbone, G., Mangialardi, L., and Mantriota, G. (2004). A Comparison of the Performances of Full and Half Toroidal Traction Drives, Mechanism and Machine Theory, Vol.39, No.9, pp. 921-42.

De Novellis, L., Carbone, G., and Mangialardi L. (2012). Traction and Efficiency Performance of the Double Roller Full-Toroidal Variator: A Comparison With Half- and Full-Toroidal Drives, Journal of Mechanical Design, Vol.134, No.7, pp. 1-14.

Faulring, E. L., Colgate, J. E., Peshkin, M. A. (2006). The Cobotic Hand Controller: Design, Control and Performance of a Novel Haptic Display, International Journal of Robotics Research, Vol. 25, No.11, pp. 1099-1119.

Grebenstein, M., Albu-Schaeffer, A., Bahls, T., Chalon, M., Eiberger, O., Friedl, W., Gruber, R., Haddadin, S., Hagn, U., Haslinger, R., Hoppner, H., Jorg, S., Nickl, M., Nothhelfer, A., Petit, F., Reill, J., Seitz, N., Wimbock, T., Wolf, S., Wusthoff, T., and Hirzinger G. (2011). The DLR Hand Arm System, In Proceedings of the IEEE International Conference on Robotics and Automation, 3175-82.

Ishida, T., and Takanashi, A.(2006). A Robot Actuator Development with High Backdrivability. In Proceedings of the IEEE International Conference on Robotics and Automation.

Ivanov, K. (2012). Self-Adjusting Motor-Wheel with CVT, International Journal of Engineering and Innovative Technology(IJEIT), Vol. 2, No. 5, pp. 287-91.

Kim, J., Park, F. C., Park, Y., and Shizuo, M. (2002). Design and Analysis of a Spherical Continuously Variable Transmission, Journal of Mechanical Design, Vol. 124, No. 1, pp. 21-29.

Li, H., Liu, W., Wang, K., Kawashima, K., and Magid, E. (2018). A Cable-Pulley Transmission Mechanism for Surgical Robot with Backdrivable Capability, Robotics and Computer-Integrated Manufacturing, Vol. 49, pp. 328-34.

Migliore, S. A., Brown, E. A., and DeWeerth, S. P.(2005). Biologically Inspired Stiffness Control, Proceedings of the IEEE International Conference on Robotics and Automation, 4508-13.

Mobedi, E., Dede, M. İ. C. (2018). A Continuously Variable Transmission (CVT) System Designed for Human-Robot Interfaces, IFToMM Asian Mechanism and Machine Science Symposium (Asian MMS 2018), Bangalore, India, 17-20 December, 2018.

Salisbury, J. K. (1980). Active Stiffness Control of a Manipulator in Cartesian Coordinates, 19th IEEE Conference on Decision and Control including the Symposium on Adaptive Processes, 95-100.

Samur, E. (2012). Performance Metrics for Haptic Interfaces. (PhD Thesis), EPFL.

Sclater, N. (2001). Mechanism \& Mechanical Devices Sourcebook, Third Edition. McGraw-Hill.

Tonietti, G., Schiavi, R., and Bicchi, A.(2005). Design and Control of a Variable Stiffness Actuator for Safe and Physical Human-Robot Interaction, In Proceedings of the IEEE International Conference on Robotics and Automation, 526-31.

Vanderborght, B., Albu-Schaeffer, A., Bicchi, A., Burdet, E., Caldwell, D.G., Carloni, R., Catalano, M., Eiberger, O., Friedl, W., Ganesh, G., Garabini, M., Grebenstein, M., Grioli, G., Haddadin, S., Hoppner, H., Jafari, A., Laffranchi, M., Lefeber, D., Petit, F., Stramigioli, S., Tsagarakis, N., Damme, M.V., Ham, R.V., Visser, L. C., and Wolf, S.(2013). Variable Impedance Actuators: A review. Robotics and Autonomous Systems, Vol. 61, No. 12, pp. 1601-14. 
Visser, L. (2013). Modeling, Control and Application to Compliant Bipedal Walking. (Master's Thesis), University of Twente.

Wolf, S., Grioli, G., Eiberger, O., Friedl, W., Grebenstein, M., Höppner, H., Burdet, E., Caldwell, D. G., Carloni, R., Catalano, M.G., Lefeber, D., Stramigioli, S., Tsagarakis, N., Damme, M.V., Ham, R. V., Vanderborght, B., Visser, L.C., Bicchi, A. and Albu-Schäffer, A. (2016). Variable Stiffness Actuators: Review on Design and Components, IEEE/ASME Transactions on Mechatronics, Vol.21, No.5, pp. 2418-30.

Wolf, S., and Hirzinger, G., (2008). A New Variable Stiffness Design: Matching Requirements of the Next Robot Generation, In Proceedings of the IEEE International Conference on Robotics and Automation, 1741-46.

Yildiz, A., and Kopmaz, O. (2015). Dynamic Analysis of a Mechanical Press Equipped with a HalfToroidal Continuously Variable Transmission, International Journal of Materials and Product Technology, Vol.50, No.1, pp. 22-36. 